Check for updates

Cite this: RSC Adv., 2017, 7, 24864

\title{
Structure-controlled CdS(OD, 1D, 2D) embedded onto 2D ZnS porous nanosheets for highly efficient photocatalytic hydrogen generation $\dagger$
}

\author{
Junmei Wang, ${ }^{\text {ab }}$ Zhijian Wang, (D) *a Li Li, ${ }^{a}$ Jiazang Chen, ${ }^{a}$ Jianfeng Zheng, ${ }^{a}$ Suping $\mathrm{Jia}^{\mathrm{a}}$ \\ and Zhenping Zhu*a
}

\begin{abstract}
The morphological characteristics of a photocatalyst is central to its photocatalytic activity for solar energy conversion. Herein, $\mathrm{Pt}-\mathrm{ZnS} / \mathrm{CdS}$ composites comprising ZnS nanosheets, embedded via the geometry and size modulation and tuning of band gap of CdS with $\mathrm{OD}, 1 \mathrm{D}$ or 2D structure, were investigated for solar hydrogen production. The photoactivity results indicate that the shape and morphology of CdS in the $\mathrm{Pt}-\mathrm{ZnS} / \mathrm{CdS}$ heterojunction play a pivotal role in affecting the photocatalytic performance. CdS with a 1D structure deposited on porous $\mathrm{Pt}-\mathrm{ZnS}$ nanosheets endow the heterojunction with increased efficiency for the separation and transport of photoinduced electron-hole pairs. The proposed mechanism for the boosted suppression of charge recombination was further confirmed by the transient photocurrent response and photoluminescence.
\end{abstract}

Received 2nd March 2017

Accepted 20th April 2017

DOI: $10.1039 / c 7 r a 02565 c$

rsc.li/rsc-advances

In this study, CdS crystals were obtained with various shapes

\section{Introduction}

Hydrogen, as a clean and sustainable energy source, has been an attractive alternative to solve the problems related to fossil fuel reserves, global climate change, and environmental pollution..$^{1-3}$ Since the pioneering work of Fujishima and Honda in $1972,{ }^{4}$ considerable efforts have been focused on preparing efficient photocatalysts in the field of water splitting for hydrogen evolution. Among all the reported semiconductor photocatalysts, CdS has been extensively studied because of its broad absorbance of visible-light and suitable conduction band position for water reduction. ${ }^{5-7}$ However, the rapid recombination of the photogenerated charge carriers and photocorrosion under light irradiation limits its wide application in photocatalysis. ${ }^{8,9} \mathrm{ZnS}$ is known as a wide band-gap semiconductor and is stable and resistant to photocorrosion..$^{10,11} \mathrm{It}$ has been reported that the coupling of ZnS with CdS greatly enhances the photocatalytic activity for hydrogen generation. ${ }^{12-14}$ Although the shape and morphology-controlled engineering of CdS, including CdS spheres, rods, and nanosheets, have been investigated, ${ }^{15-17}$ a systematic study on their hybridization with $\mathrm{ZnS}$ and the heterojunction-related band potential alignment for photocatalysis applications is still lacking.

${ }^{a}$ State Key Library of Coal Conversion, Institute of Coal Chemistry, Chinese Academy of Science, Taiyuan, 03001, P. R. China.E-mail:wangzhijian@sxicc.ac.cn; zpzhu@sxicc. ac.cn

${ }^{b}$ University of Chinese Academy of Science, Beijing, 100049, P. R. China

$\dagger$ Electronic supplementary information (ESI) available. See DOI: $10.1039 / \mathrm{c} 7 \mathrm{ra02565 \textrm {c }}$ from QDs, with 1D to 2D structures, by simply adjusting the reflux reaction temperature. The shape selective growth of CdS was hybridized with a porous $\mathrm{ZnS}$ nanosheet support to form a type-II heterojunction via a Fermi-level alignment with a Pt metal co-catalyst first pre-deposited on the ZnS surface. This fabrication enhanced both the efficiency and stability of the composite for photocatalytic hydrogen evolution. In addition, the porous ZnS nanosheets possess a high specific surface area and large pore volume, providing more active reaction sites for water reduction and facilitating the charge transfer through the nanoporous structure. ${ }^{18,19}$ Herein, we report a comparative study on the composites of a porous ZnS support coupled with different morphologies of CdS in terms of their hydrogen production under visible light irradiation. The results showed that the CdS nanorod-modified porous ZnS nanosheets displayed the highest photocatalytic activity of about $26 \mathrm{mmol} \mathrm{g}^{-1}$ $\mathrm{h}^{-1}$ for hydrogen production.

This visible light responsive heterojunction composite is expected to provide some inspiration for the fabrication of efficient photocatalyst hybrids via control of the shape and size of the semiconductor crystals to modulate the band structure towards optimizing their associated photocatalytic performance.

\section{Experimental}

\section{Synthesis of ZnS}

The $\mathrm{ZnS}$ nanoporous sheets were synthesized using a reflux method. $\mathrm{Zn}(\mathrm{Ac})_{2} \cdot 2 \mathrm{H}_{2} \mathrm{O}(28 \mathrm{mmol})$ and thiourea $(56 \mathrm{mmol})$ were dissolved in $30 \mathrm{~mL}$ of $\mathrm{H}_{2} \mathrm{O}$ and $40 \mathrm{~mL}$ of ethylenediamine. The 
mixture was refluxed and stirred for $13 \mathrm{~h}$ at $110{ }^{\circ} \mathrm{C}$. After the reaction, the resulting white product was obtained by centrifugation and washed several times with deionized water and absolute ethanol. Then, the sample was dried in vacuum at $60{ }^{\circ} \mathrm{C}$ for $24 \mathrm{~h}$.

\section{Synthesis of CdS}

The CdS QDs were synthesized using a reflux method. $\mathrm{Cd}(\mathrm{Ac})_{2} \cdot 2 \mathrm{H}_{2} \mathrm{O}(28 \mathrm{mmol})$ and thiourea $(56 \mathrm{mmol})$ were dissolved in $30 \mathrm{~mL}$ of deionized water and $64 \mathrm{~mL}$ of diethylenetriamine (DETA). The mixture was refluxed and stirred at $25{ }^{\circ} \mathrm{C}$ for $13 \mathrm{~h}$. After the reaction, the resulting yellow product was obtained by centrifugation and washed several times with deionized water and absolute ethanol. Then, the sample was dried in vacuum at $60{ }^{\circ} \mathrm{C}$ for $24 \mathrm{~h}$. The syntheses of the CdS $2 \mathrm{D}$ and CdS 1D composites were conducted under the same procedure except at the reflux temperature of $90^{\circ} \mathrm{C}$ and $120^{\circ} \mathrm{C}$, respectively.

\section{Photodeposition of Pt-ZnS}

Herein, $1 \mathrm{~g}$ of the obtained $\mathrm{ZnS}$ powder was suspended in 230 $\mathrm{mL}$ of a methanol/water solution $(10 \% \mathrm{v} / \mathrm{v})$ containing $\mathrm{H}_{2}$ $\mathrm{PtCl}_{6} \cdot \mathrm{H}_{2} \mathrm{O}(2 \mathrm{wt} \% \mathrm{Pt} v$ s. $\mathrm{ZnS}$ ) under stirring for $0.5 \mathrm{~h}$ and then exposed to UV light for $3 \mathrm{~h}$. After the photodeposition step, the products were obtained by centrifugation and washed several times with deionized water and then dried in vacuum at $80{ }^{\circ} \mathrm{C}$ for $24 \mathrm{~h}$.

\section{Synthesis of Pt-ZnS/CdS}

The binary Pt-ZnS/CdS QDs composites were prepared using a reflux method. Typically, $0.1 \mathrm{~g}$ of the prepared $\mathrm{Pt}-\mathrm{ZnS}$ was dispersed in a mixture containing $30 \mathrm{~mL}$ of deionized water and $64 \mathrm{~mL}$ of diethylenetriamine (DETA). $\mathrm{Cd}(\mathrm{Ac})_{2} \cdot 2 \mathrm{H}_{2} \mathrm{O}(28 \mathrm{mmol})$ and thiourea $(56 \mathrm{mmol})$ were added into the solution to form a suspension, which was refluxed at $15{ }^{\circ} \mathrm{C}$ for $13 \mathrm{~h}$. The composite was obtained and washed several times with water and then ethanol and finally dried in vacuum at $60{ }^{\circ} \mathrm{C}$ for $24 \mathrm{~h}$. The final composite powder weighed $0.49 \mathrm{~g}$. The syntheses of the Pt-ZnS/CdS 2D and Pt-ZnS/CdS 1D composites were conducted using the same procedure but varying the reflux temperature to $90{ }^{\circ} \mathrm{C}$ and $120{ }^{\circ} \mathrm{C}$, respectively, and the final composites weighed $0.48 \mathrm{~g}$ and $0.50 \mathrm{~g}$, respectively.

\section{Characterization}

Transmission electron microscopy images were obtained using a high-resolution transmission electron microscope (JEM-2100, $200 \mathrm{kV}$ ). X-ray diffraction (XRD) was performed using a Bruker D8 Advance X-ray powder diffractometer with $\mathrm{Cu} \mathrm{K} \alpha(\lambda=1.5406$ $\AA)$ radiation. The morphologies of the photocatalysts were investigated via field emission scanning electron microscopy (JSM-7001F, operated at $10 \mathrm{kV}$ ). UV-visible absorption spectroscopy was carried out using a Shimadzu UV-3600 UV-Vis-NIR spectrophotometer at room temperature. $\mathrm{BaSO}_{4}$ was used as the reflectance standard. The photoluminescence was measured using a fluorescence spectrometer (F-7000, Hitachi, Japan). X- ray photoelectron spectroscopy (XPS) and ultraviolet photoelectron spectroscopy (UPS) were carried out using a Thermo ESCALAB 250 XPS spectrometer. The effect of the sample surface charge was eliminated by shifting the XPS peak of carbon C 1 s to $284.8 \mathrm{eV}$.

\section{Photocatalytic measurements}

The photocatalytic reactions were carried out in a flowing system (Ar gas with a flow rate at $1.5 \mathrm{~L} \mathrm{~h}^{-1}$ ) with an innerirradiation-type pyrex reactor and a $300 \mathrm{~W}$ xenon arc light source after filtering the UV light using a quartz water jacket filling with a chilled circulating $\mathrm{NaNO}_{2}$ aqueous solution $(1 \mathrm{M})$ to pass only visible light $(\lambda>400 \mathrm{~nm}) .^{20,21}$ The recycling $\mathrm{NaNO}_{2}$ solution was also utilized to maintain a constant reactor temperature at $298 \mathrm{~K}$. Then, $50 \mathrm{mg}$ of the photocatalyst was suspended in $230 \mathrm{~mL}$ lactic acid aqueous solution $(20 \mathrm{~mL}$ lactic acid, $210 \mathrm{~mL}$ water). Prior to irradiation, the reactor was purged with Ar gas for $25 \mathrm{~min}$ to replace the air inside the solution. Then, $1 \mathrm{~mL}$ of gas was intermittently sampled from the flowing system, and then, the amount of $\mathrm{H}_{2}$ evolved was analyzed by gas chromatography (Beifen-Ruili:SP-2100. MS-5A column. TCD. Ar carrier).

\section{Electrochemical measurements}

The photocurrent measurements were performed via an electrochemical workstation (CHI 660D Shanghai, China) using a standard three-electrode system with the prepared samples as the working electrode, a Pt foil as the counter electrode, and a standard calomel electrode in saturated $\mathrm{KCl}$ as the reference electrode. The electrolyte was an aqueous solution containing $0.25 \mathrm{mM} \mathrm{Na}_{2} \mathrm{~S}$ and $0.25 \mathrm{mM} \mathrm{Na}_{2} \mathrm{SO}_{3}$. The working electrode was prepared as follows: the photocatalyst $(0.1 \mathrm{~g})$ was stirred with 3 $\mathrm{mL}$ of isopropyl alcohol for $24 \mathrm{~h}$ to form a slurry, and then, the slurry was spin-coated onto FTO glass for further investigation.

\section{Results and discussion}

The as-synthesized ZnS shows a uniform porous 2D sheet-like morphology with a large lateral dimension of several hundreds of nanometers and width of about $150 \mathrm{~nm}$ (Fig. 1a).

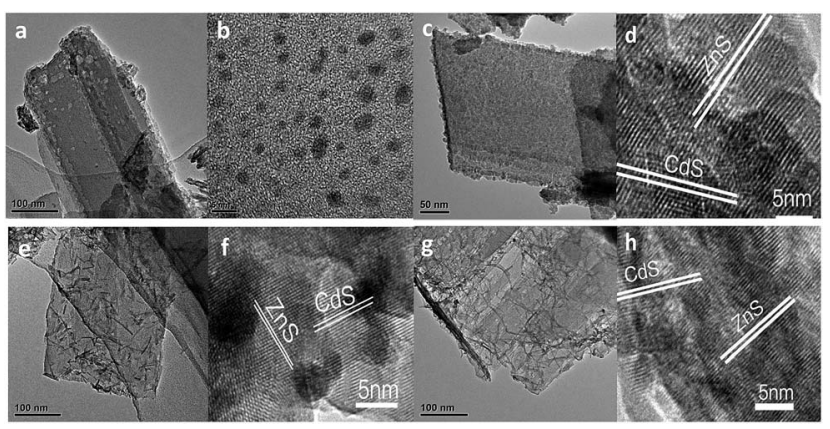

Fig. 1 TEM images of Pt-ZnS (a), CdS QDs (b), Pt-ZnS/CdS QDs (c), $\mathrm{Pt}-\mathrm{ZnS} / \mathrm{CdS} 1 \mathrm{D}(\mathrm{e})$ and $\mathrm{Pt}-\mathrm{ZnS} / \mathrm{CdS} 2 \mathrm{D}(\mathrm{g})$, and the high resolution TEM images of Pt-ZnS/CdS QDs (d), Pt-ZnS/CdS 1D (f) and Pt-ZnS/ CdS 2D (h). 
All the Pt-ZnS/CdS composites exhibited a plate morphology constructed by the ZnS plates and CdS with shapes of nanoparticles, sheets, and nanorods. As shown in Fig. 1b, the CdS quantum dots were uniformly dispersed with the diameters of about $2 \mathrm{~nm}$. Fig. 1c shows the Pt-ZnS/CdS QDs composites. The CdS quantum dots with an average diameter of $2 \mathrm{~nm}$ were closely embedded into the pores distributed in the ZnS plates. Fig. 1e shows the Pt-ZnS/CdS1D composites. The ZnS plates were covered by the $1 \mathrm{D}$ CdS nanorods with a uniform size of around $10 \mathrm{~nm}$ width and $90 \mathrm{~nm}$ length. Especially, the CdS 2D nanosheets with very low thickness were firmly attached to the ZnS surface, as shown in Fig. 1g. Fig. 1d, f, and h show the high resolution TEM images of Pt-ZnS/CdS QDs, Pt-ZnS/CdS 1D, and $\mathrm{Pt}-\mathrm{ZnS} / \mathrm{CdS} 2 \mathrm{D}$, respectively. We can clearly see the crystal lattice of CdS and $\mathrm{ZnS}$ for all the samples. The TEM images of the photocatalyst showed that ZnS and CdS closely interacted with each other to form a uniform composite, as shown in Fig. S1. $\dagger$ To further determine the structure of the Pt-ZnS/CdS composites, the X-ray diffraction (XRD) patterns were obtained, as shown in Fig. 2. The broadened peak observed at about $20^{\circ}$ for Pt-ZnS/CdS QDs can be ascribed to the diffraction of cubic CdS with small sizes. It was found that the peak intensity of the Pt-ZnS/CdS 2D composite corresponding to the cubic phase was weak when compared with that of the Pt-ZnS/ CdS QDs, which can be attributed to the CdS 2D constituent preserving the structure and morphology of small and thin sheets with wrinkles. The sharp and narrow peaks in the XRD spectra of the Pt-ZnS/CdS 1D composite suggest that the CdS nanorods in the composites were well-crystallized with a hexagonal phase. The $\mathrm{ZnS}$ diffraction peak was not obvious for all the samples due to being covered and surrounded by the CdS crystals.

X-ray photoelectron spectroscopy was employed to analyze the chemical composition of the photocatalysts. As shown in Fig. S2, $\dagger$ the typical Cd 3d peaks at 405.2 and $411.9 \mathrm{eV}$ and $\mathrm{S} 2 \mathrm{p}$ peaks at 161.5 and $162.7 \mathrm{eV}$ corresponding to $\mathrm{CdS}$ could be clearly seen. The peaks of $\mathrm{Zn} 2 \mathrm{p}$ located at about 1044.2 and 1021.3 eV, implying the ZnS form, were also observed. The

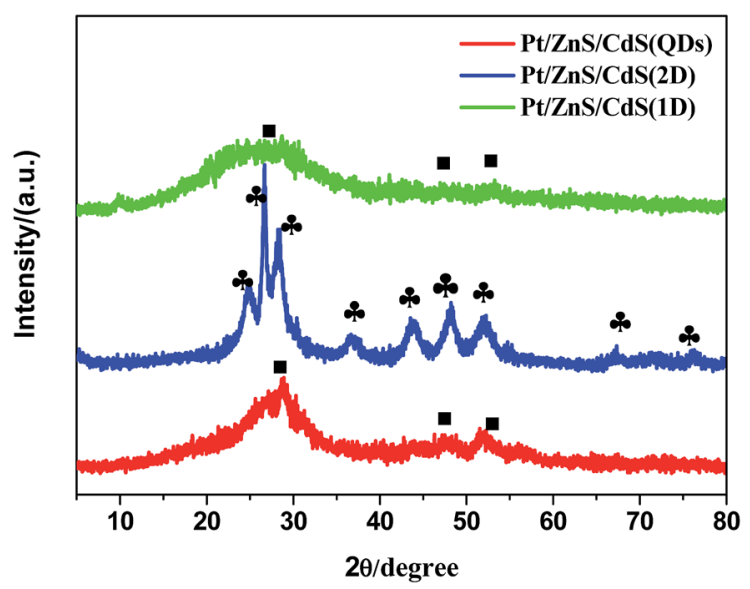

Fig. 2 The XRD patterns of Pt-ZnS/CdS QDs, Pt-ZnS/CdS 1D and Pt$\mathrm{ZnS} / \mathrm{CdS} 2 \mathrm{D}$ (ם-corresponds to cubic CdS, a-corresponds to hexagonal CdS). survey scan implied that the compounds were closely fabricated with high purity.

$\mathrm{N}_{2}$ adsorption-desorption isotherms and corresponding $\mathrm{BJH}$ (Barret-Joyner-Halenda) analysis were employed to determine the specific surface area and pore size distribution of the PtZnS/CdS composites, respectively. As shown in Fig. 3, the PtZnS/CdS 1D and Pt-ZnS/CdS 2D exhibited a higher BET surface area of $65 \mathrm{~m}^{2} \mathrm{~g}^{-1}$ and $77 \mathrm{~m}^{2} \mathrm{~g}^{-1}$, respectively, which was much larger than that of the Pt-ZnS/CdS QDs $\left(48 \mathrm{~m}^{2} \mathrm{~g}^{-1}\right)$. The results indicated that the porous $\mathrm{ZnS}$ were filled with the tiny CdS QDs. Based on the BJH analysis of the desorption branch of the isotherm, the pore diameter increased with the increase in the dimensions of CdS(0D-1D-2D). The pore size of the Pt-ZnS/CdS QDs were mainly distributed at about $11 \mathrm{~nm}$ due to the occupation of the mesopores by the CdS QDs. However, the proportion of pores larger than $20 \mathrm{~nm}$ clearly grew for $\mathrm{Pt}-\mathrm{ZnS} /$ CdS 2D, which was attributed to the face to face aggregation of the $\mathrm{ZnS} 2 \mathrm{D}$ and $\mathrm{CdS} 2 \mathrm{D}$ combination. A comparison of the UV-Vis diffuse reflectance spectra of Pt-ZnS/CdS QDs, Pt-ZnS/ CdS 1D, and Pt-ZnS/CdS $2 \mathrm{D}$ is displayed in Fig. 4a. The absorption edges of Pt-ZnS/CdS QDs, Pt-ZnS/CdS 1D, and Pt$\mathrm{ZnS} / \mathrm{CdS} 2 \mathrm{D}$ were estimated to be $520 \mathrm{~nm}, 470 \mathrm{~nm}$, and $460 \mathrm{~nm}$, respectively, which showed red shifts with the decrease in the CdS dimensions (2D nanosheets, 1D nanorods to 0D nanoparticles). The corresponding energy band gaps of the samples can be obtained via the plots of $(\alpha h \nu)^{2} v s$. $h \nu$, as shown in Fig. $4 \mathrm{~b}$. The band gap value of CdS with $0 \mathrm{D}, 1 \mathrm{D}$, and $2 \mathrm{D}$ shapes were estimated to be $2.45 \mathrm{eV}, 2.65 \mathrm{eV}$, and $2.69 \mathrm{eV}$, respectively, in the composites. To further calculate the band structure of the composites, UPS measurements were conducted to determine the valence band potentials of the Pt-ZnS/CdS composites. The UPS results showed that the valence-band maximum (VBM) of CdS (0D-1D-2D) was about $1.83 \mathrm{eV}, 1.69 \mathrm{eV}$, and $1.95 \mathrm{eV}$, respectively (Fig. 5). The results illustrated that the conduction
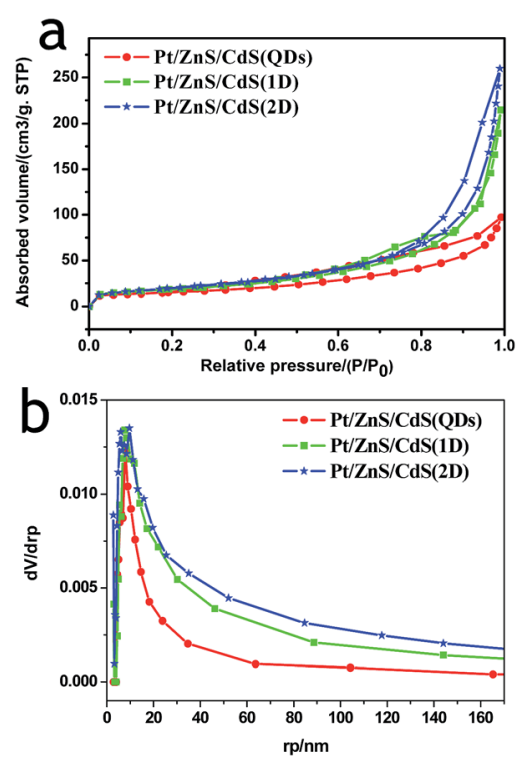

Fig. $3 \quad \mathrm{~N}_{2}$ adsorption-desorption isotherms (a) and corresponding $\mathrm{BJH}$ pore size distribution curve calculated from the adsorption isotherm of Pt-ZnS/CdS QDs, Pt-ZnS/CdS 1D and Pt-ZnS/CdS 2D (b). 

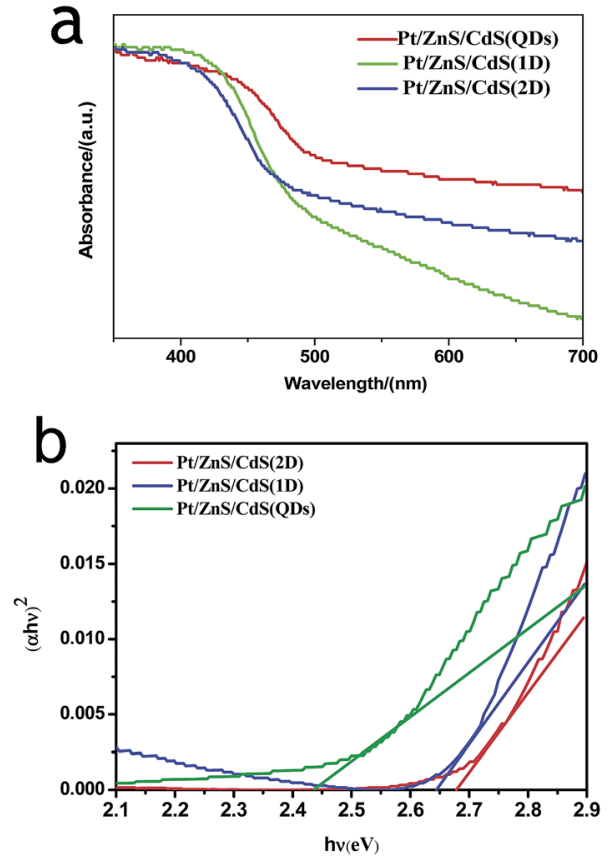

Fig. 4 The light absorbance spectra (a) and Tauc plots (b) of Pt-ZnS/ CdS QDs, Pt-ZnS/CdS 1D and Pt-ZnS/CdS 2D.

band values can be estimated to be $-0.62 \mathrm{eV},-0.96 \mathrm{eV}$, and $-0.74 \mathrm{eV}$, respectively. The calculated band energy levels are shown in Fig. 6.

To ensure whether the morphological characteristics of CdS with $0 \mathrm{D}, 1 \mathrm{D}$, and $2 \mathrm{D}$ supported on the $2 \mathrm{D} \mathrm{ZnS}$ nanosheets have any effect on the photocatalytic activity in a Pt-ZnS/CdS heterojunction system, we conducted $\mathrm{H}_{2}$ generation experiments. As shown in Fig. 7a and b, the $\mathrm{H}_{2}$ production rate of Pt-ZnS/CdS QDs was $7.7 \mathrm{mmol} \mathrm{g}^{-1} \mathrm{~h}^{-1}$; when the CdS QDs in the composites was replaced with $\mathrm{CdS} 2 \mathrm{D}$, the $\mathrm{H}_{2}$ production rate was significantly enhanced to $21 \mathrm{mmol} \mathrm{g}^{-1} \mathrm{~h}^{-1}$. The highest activity was obtained with the Pt-ZnS/CdS 1D system, reaching to about $26 \mathrm{mmol} \mathrm{g}^{-1} \mathrm{~h}^{-1}$. Based on the abovementioned structure, the band gap energy, and BET analysis, which are shown in Table S1, $\uparrow$ we attributed the good performance to the following three features. First, with the same cubic CdS crystal structure, the specific surface area of Pt-ZnS/CdS 2D was larger

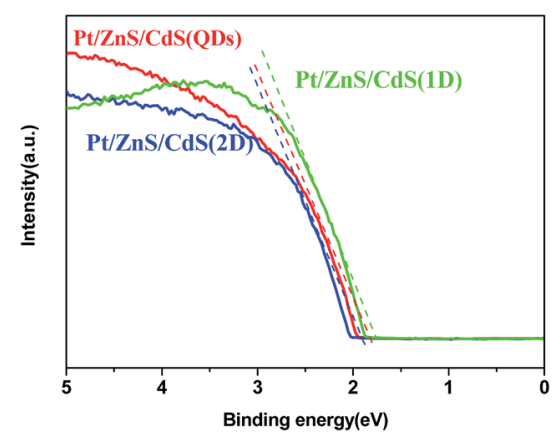

Fig. 5 The UPS spectrum of Pt-ZnS/CdS QDs, Pt-ZnS/CdS 1D, and $\mathrm{Pt}-\mathrm{ZnS} / \mathrm{CdS} 2 \mathrm{D}$.

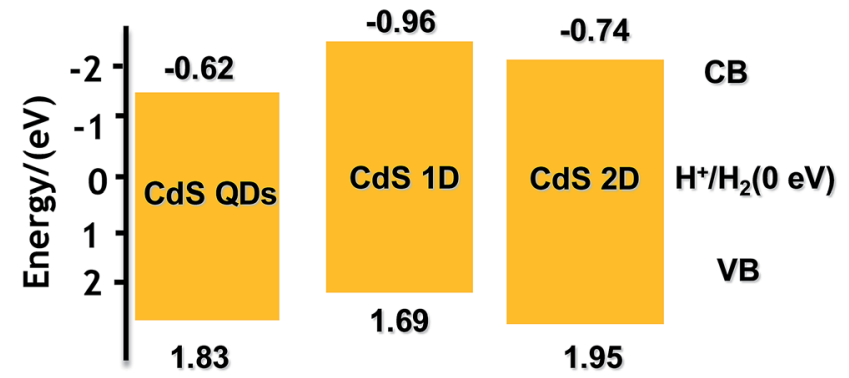

Fig. 6 The band structure alignments of $\mathrm{CdS}$ in Pt-ZnS/CdS QDs, PtZnS/CdS 2D, and Pt-ZnS/CdS 1D.

than that of Pt-ZnS/CdS QDs $\left(77 \mathrm{~m}^{2} \mathrm{~g}^{-1}\right.$ vs. $\left.48 \mathrm{~m}^{2} \mathrm{~g}^{-1}\right)$, which means that there were more active sites for the photocatalytic reduction to occur at and the more porous nanostructure provided efficient mass-transport pathways. Second, when compared to Pt-ZnS/CdS 2D, Pt-ZnS/CdS 1D had a smaller specific surface area $\left(77 \mathrm{~m}^{2} \mathrm{~g}^{-1}\right.$ vs. $\left.65 \mathrm{~m}^{2} \mathrm{~g}^{-1}\right)$, whereas the CdS 1D with hexagonal crystal structure played a predominant role in enhancing the photoactivity rather than the cubic CdS 2D in the Pt-ZnS/CdS 2D heterojunction. Additionally, the nanorod crystals with the special lateral 1D geometry are beneficial for the transport of the excited electrons to the Pt co-catalyst supported on the 2D ZnS nanosheets. Third, based on the band energy diagrams shown in Fig. 6, the band structure of the CdS 1D in the Pt-ZnS/CdS 1D heterojunction has the best advantage for charge transfer from the CdS 1D sensitizer to the deposited Pt, which plays a key role in the photocatalytic $\mathrm{H}_{2}$ generation performance. The process of the photogenerated charge carriers is schematically shown in Fig. 8. To evaluate the stability of the $\mathrm{Pt}-\mathrm{ZnS} / \mathrm{CdS}$ 1D photocatalyst, a recycling test was performed. As shown in Fig. 9, no significant decrease in the $\mathrm{H}_{2}$ production
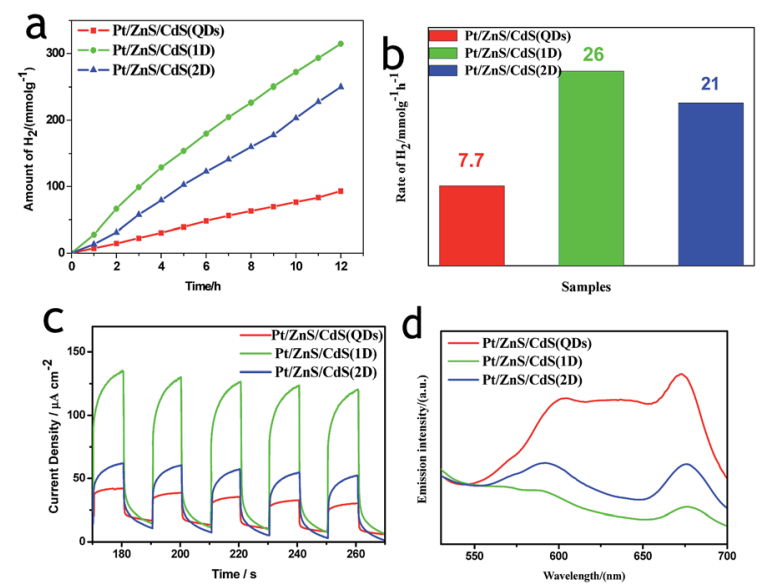

Fig. 7 ( $a$ and $b$ ) A comparison of the $\mathrm{H}_{2}$ evolution activity of Pt-ZnS/ CdS QDs, Pt-ZnS/CdS 1D, and Pt-ZnS/CdS 2D, respectively. Light source: $300 \mathrm{~W}$ Xe lamp ( $\lambda>400 \mathrm{~nm}$ ). Reaction: $0.05 \mathrm{~g}$ of catalyst dispersed in $230 \mathrm{~mL}$ of deionized water containing lactic acid $(10 \% \mathrm{v} / \mathrm{v})$. (c) The photocurrent response in an $\mathrm{Na}_{2} \mathrm{~S}_{2} \mathrm{Na}_{2} \mathrm{SO}_{3}$ aqueous solution with on-off visible light illumination $\left(100 \mathrm{~mW} \mathrm{~cm}^{-2}\right)$ at $0.0 \mathrm{~V}$ vs. NHE. (d) The photoluminescence spectra of Pt-ZnS/CdS QDs, Pt-ZnS/ CdS1D, and Pt-ZnS/CdS 2D. 

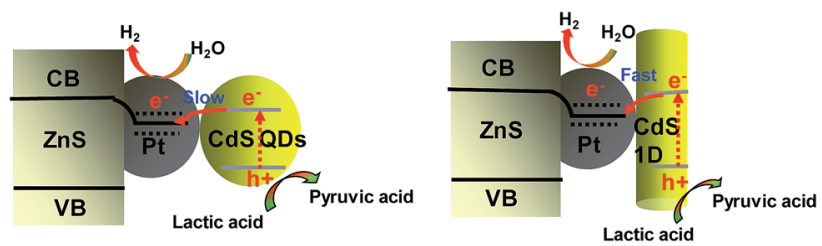

Fig. 8 A schematic of the charge separation and electron transfer in the $\mathrm{Pt}-\mathrm{ZnS} / \mathrm{CdS}$ system with different fabrication under visible light irradiation.

was observed after 5 cycles, which indicates good stability of the $\mathrm{Pt}-\mathrm{ZnS} / \mathrm{CdS}$ 1D composite against photocorrosion. After the stability test, the composite was obtained and further characterized by XRD, XPS, and TEM, as shown in Fig. S3, $\dagger$ and all the results show no obvious changes when compared with those of the original catalysts.

Note that all the novel binary $\mathrm{Pt}-\mathrm{ZnS} / \mathrm{CdS}$ porous composite photocatalysts display a higher $\mathrm{H}_{2}$ production rate compared with the corresponding CdS catalysts, as shown in Fig. S5. $\dagger$ The combined heterojunction is beneficial to improve the efficiency of CdS in the photocatalytic $\mathrm{H}_{2}$ evolution reaction. The porous $\mathrm{Pt}-\mathrm{ZnS}$ functions as a template for the fabrication of $\mathrm{Pt}-\mathrm{ZnS} /$ CdS. A type I heterojunction was formed by compounding the wide band gap semiconductor of $\mathrm{ZnS}$ with the narrow band gap semiconductor of CdS. This combination can effectively protect the CdS from photocorrosion..$^{\mathbf{1 4 2 2}}$ It has been demonstrated that there are many intrinsic defects such as zinc vacancies $\left(V_{\mathrm{Zn}}\right)$ and interstitial sulfur vacancies $\left(I_{\mathrm{s}}\right)$ in the $\mathrm{ZnS}$ porous structure. $V_{\mathrm{Zn}}$ and $I_{\mathrm{S}}$ can act as acceptors for the holes generated in the CdS NRs, ${ }^{13,23}$ which can enhance the stability of CdS. On the other hand, as can be seen in the Fig. 1, CdS was uniformly and tightly dispersed on the surface of $\mathrm{ZnS}$, and the porous $\mathrm{ZnS}$ structure adsorbed more sacrificial agent of lactic acid, which is beneficial for trapping the holes generated on the CdS surface. The advantages of the intrinsic defects and porous structure of $\mathrm{ZnS}$ can enhance the efficiency and stability of Pt-ZnS/CdS in photocatalytic $\mathrm{H}_{2}$ evolution.

To verify the displayed photocatalytic mechanism, the transient photocurrent response of the samples was investigated, and the results are shown in Fig. 7c. The photocurrent value of $\mathrm{Pt}-\mathrm{ZnS} / \mathrm{CdS} 2 \mathrm{D}$ was higher than that of Pt-ZnS/CdS QDs, and a significantly enhanced photocurrent value was observed with

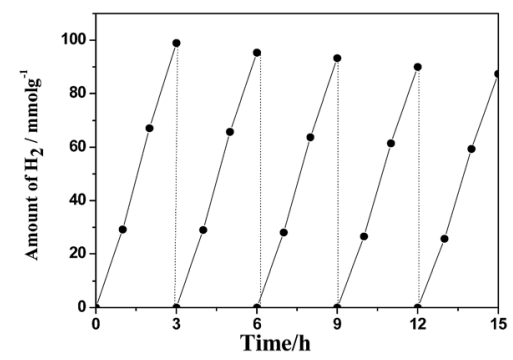

Fig. 9 The cycle test on the Pt-ZnS/CdS 1D composite suspended in $230 \mathrm{~mL}$ of water containing lactic acid $(10 \% \mathrm{v} / \mathrm{v})$ aqueous solution bubbled with Ar for $3 \mathrm{~h}$ under visible light irradiation.

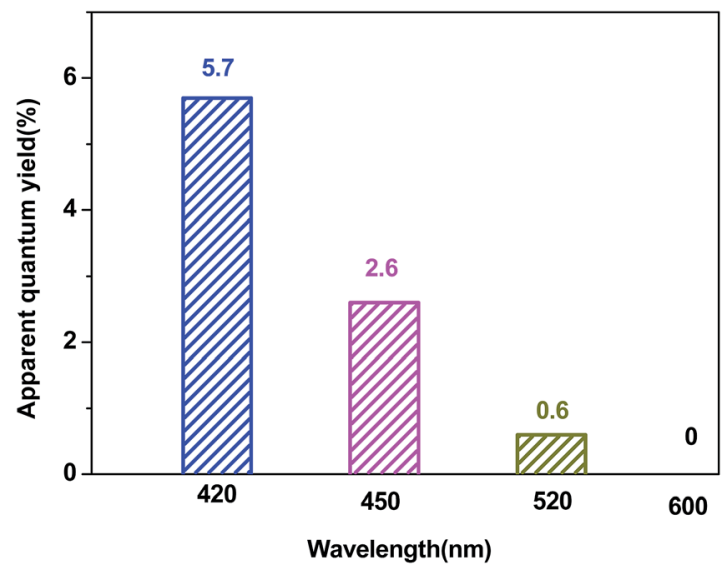

Fig. 10 A comparison of the apparent quantum yield of the Pt-ZnS/ CdS 1D composite suspended in a lactic acid (10\% v/v) aqueous solution under irradiation at different wavelengths.

the Pt-ZnS/CdS 1D composite. As the band energy gap of CdS $1 \mathrm{D}$ is wider when compared to that of the CdS QDs and approximate to that of CdS 2D, the high photocurrent value of $\mathrm{Pt}-\mathrm{ZnS} / \mathrm{CdS}$ 1D was mainly attributed to the following three main reasons: the improved charge transport from CdS $1 \mathrm{D}$ to $\mathrm{Pt}-\mathrm{ZnS}$, good heterojunction formation of $\mathrm{Pt}-\mathrm{ZnS} / \mathrm{CdS}$ 1D composites, and the promoted separation of the electron-hole pairs generated in the CdS 1D sensitizer. The photoluminescence (PL) spectra were obtained to confirm the migration, transfer, and recombination process of the photogenerated electron-hole charge carriers. As shown in Fig. 7d, the emission peaks were at about $595 \mathrm{~nm}$ and $675 \mathrm{~nm}$. The emission intensity of Pt-ZnS/CdS 2D was drastically quenched when compared with that of Pt-ZnS/CdS QDs, indicating that the CdS 2D in the Pt-ZnS/CdS 2D heterojunctions can transfer the photogenerated electrons and holes to different sides of the composites, thus suppressing their recombination. The PL intensity of Pt-ZnS/CdS 1D further diminished when compared with that of $\mathrm{Pt}-\mathrm{ZnS} / \mathrm{CdS} 2 \mathrm{D}$, which was attributed to the $1 \mathrm{D}$ geometry structure and hexagonal crystallinity of the CdS 1D. The 1D structure can boost the separation and transfer of the photogenerated electron-hole pairs and eventually decrease the recombination process. The result was consistent with the observation in photoelectrochemistry and photocatalytic activity for the $\mathrm{H}_{2}$ evolution experiment.

Fig. 10 shows the apparent quantum yield of hydrogen production as a function of the wavelength of the incident light. The apparent quantum efficiency was measured under similar conditions, except for the light intensity and the wavelength regions of the irradiation light. A set of glass filters was adopted to control the wavelength regions of incident light. The quantum efficiency decreased as the wavelength increased.

\section{Conclusions}

Novel binary Pt-ZnS/CdS porous composite photocatalysts with various shapes of CdS embedded onto $\mathrm{ZnS}$ sheets were fabricated using a facile reflux process. The highest photocatalytic $\mathrm{H}_{2}$ 
production performance was obtained on the one-dimensional CdS nanorod-sensitized porous Pt-ZnS nanosheet heterojunction. The excellent photocatalytic activity for hydrogen evolution from water using lactic acid as a sacrificial reagent under visible light irradiation arised from the combined effects between the layered mesoporous $\mathrm{ZnS}$ support and CdS nanorods. This novel configuration can efficiently suppress the charge recombination by the spatial separation of photoexcited electrons on Pt-ZnS sheets and the holes left on the CdS. The existence of mesoporous nanosheets and special nanorods facilitate charge separation and transfer at the interface of the heterojunction constructed between the Pt-ZnS and CdS.

In particular, the photocatalyst shows good stability and anti-photocorrosion under visible-light irradiation. This study demonstrates, for the first time, that the heterojunction configuration and associated catalytic efficiency can be optimized by adjusting the crystal structure and morphology of the catalysts. The morphology-controlled synthesis technique can provide a way for designing other high performance heterojunction photocatalysts.

\section{Acknowledgements}

We acknowledge the financial support received from the National Natural Science Foundation of China (No. U1510108 and 91545116).

\section{References}

1 A. Tanaka, K. Teramura, S. Hosokawa, H. Kominam and T. Tanaka, Chem. Sci., 2017, 8, 2574.

2 K. Chang, Z. Mei, T. Wang, Q. Kang, S. Ouyang and J. Ye, ACS Nano, 2014, 8, 7078.

3 Y. Hou, B. Abrams, P. Vesborg, M. Björketun, K. Herbst, L. Bech, A. Setti, C. Damsgaard, T. Pedersen, O. ansen, J. Rossmeisl, S. Dahl, J. Nørskov and I. Chorkendorff, Nat. Mater., 2011, 10, 434.

4 A. Fujishima and K. Honda, Nature, 1972, 238, 37.
5 K. Zhang, W. Kim, M. Ma, X. Shi and J. Park, J. Mater. Chem. A, 2015, 3, 4803.

6 N. Bao, L. Shen, T. Takata and K. Domen, Chem. Mater., 2008, 39, 110.

7 H. Yu, X. Huang, P. Wang and J. Yu, J. Phys. Chem. C, 2016, 120, 3722 .

8 P. Du, Y. Zhu, J. Zhang, D. Xu, W. Peng, G. Zhang, F. Zhang and X. Fan, RSC Adv., 2016, 6, 74394.

9 R. Marschall, Adv. Funct. Mater., 2014, 24, 2421.

10 G. Chen, F. Li, Y. Fan, Y. Luo, D. Li and Q. Meng, Catal. Commun., 2013, 40, 51.

11 A. Hernández-Gordillo, F. Tzompantzi and R. Gómez, Int. J. Hydrogen Energy, 2012, 37, 17002.

12 T. Zhuang, Y. Liu, M. Sun, S. Jiang, M. Zhang, X. Wang, Q. Zhang, J. Jiang and S. Yu, Angew. Chem., Int. Ed., 2015, 127, 11446.

13 Y. Xie, Z. Yu, G. Liu, X. Ma and H. Cheng, Energy Environ. Sci., 2014, 7, 1895.

14 L. Huang, X. Wang, J. Yang, G. Liu, J. Han and C. Li, J. Phys. Chem. C, 2013, 117, 11584.

15 H. Kim, T. Kim, I. Kim and S. Hwang, Adv. Funct. Mater., 2011, 21, 3111.

16 Y. Xu, W. Zhao, R. Xu, Y. Shi and B. Zhan, Chem. Commun., 2013, 49, 9803.

17 Z. Tang, B. Han, C. Han and Y. Xu, J. Mater. Chem. A, 2017, 5, 2387.

18 Y. Li, C. Gang, Q. Wang, A. Zhou and Z. Shen, Adv. Funct. Mater., 2010, 20, 3390.

19 J. Jang, C. Yu, S. Choi, S. Ji, E. Kim and J. Lee, J. Catal., 2008, 254, 144.

20 K. Maeda, T. Takata, M. Hara, N. Saito, Y. Inoue, H. Kobayashi and K. Domen, J. Am. Chem. Soc., 2005, 127, 8286.

21 P. Yang, J. Zhao, J. Wang, B. Cao, L. Li and Z. Zhu, J. Mater. Chem. A, 2014, 3, 136.

22 D. Jiang, Z. Sun, H. Jia, D. Lu and P. Du, J. Mater. Chem. A, 2016, 4, 675 .

23 G. Wang, B. Huang, Z. Li, Z. Lou, Z. Wang, Y. Dai and M. Whangbo, Sci. Rep., 2015, 5(4), 8544. 